\title{
AFFINE INVARIANT DESCRIPTORS OF 3D OBJECT USING MULTIPLE REGRESSION MODEL
}

\author{
M. Elhachloufi ${ }^{1}$, A. El Oirrak ${ }^{1}$, D. Aboutajdine ${ }^{2}$, M.N. Kaddioui ${ }^{1}$ \\ ${ }^{1}$ Faculty Semlalia, Department of Informatics, Marrakech, Morocco \\ elhachloufi@yahoo.fr, oirrek@yahoo.fr,kaddioui@ucam.ac.ma \\ ${ }^{2}$ Faculty of Science, LEESA-GSCM, BP 1014, RABAT \\ aboutajefsr.ac.ma
}

\begin{abstract}
In this work, a new method invariant [1,2,3] for 3D object is proposed using multiple regression model. This method consists of extracting an invariant vector using the multiple linear parameters model applied to the 3D object, it's invariant against affine transformation of this object.

The concerned $3 D$ objects are transformations of $3 D$ objects by one element of the overall transformation. The set of transformations considered in this work is the general affine group.
\end{abstract}

\section{KEY WORDS}

Invariant, 3D Object, multiple regression model, Affine Transformation

\section{INTRODUCTION}

With the advent of the Internet, exchanges and the acquisition of information, description and recognition of $3 \mathrm{D}$ objects have been as extensive and have become very important in several domains.

On the other hand, the size of 3D objects used on the Internet and in computer systems has become enormous, particularly due to the rapid advancement technology acquisition and storage which require the establishment of methods to develop description and recognition techniques $[4,5,6]$ to access intelligently to the contents of these objects.

For this, shape descriptors[7] can be used to provide a unique description, compact and meaningful content of a 3D object. These descriptors should check the properties of invariance to a class of transformations, discrimination, stability and completeness [8].

In the literature, several descriptors have been proposed for 3D objects. In the context of the standard MPEG7, a 3D object is described by a set of two-dimensional primitives (2D). These primitives are calculated using different projections of the object obtained by considering different angles of views. The effectiveness of these methods of description called 2D/3D depends on the number of these projections [9].

Other methods have proposed 3D shape descriptors[10] computed from a volumetric representation associated with the surface representation of the object. We cite in particular, the descriptors using the 3D Fourier transform [11], 3D moments [12,13] and harmonic analysis $[14,15]$. Moreover, a 3D shape descriptor intrinsically linked to the local geometry of the surface of a 3D object was proposed in [16]. The shape spectrum descriptor called 3D (3D 
SSD) is invariant to geometric transformations. However, it is not robust to topological changes. The optimal 3D Hough Descriptor (DH3DO) has been proposed to remedy this drawback [17].

In this context, our work consists of presenting an invariant method for 3D objects using linear regression model.

Our work is organized as follows: section 1 concerns the representation of 3D objects. The multiple linear models are presented in section 2 . In section 3, we propose the method for affine invariant. Then, in section 4, experimental results are presented and discussed.

\section{REPRESENTATION OF THE 3D OBJECT}

3D object is represented by a set of points denoted $M=\left\{P_{i}\right\}_{i=1 \ldots \ldots . . n} \quad$ where $P_{i}=\left(x_{i}, y_{j}, z_{i}\right) \in \mathfrak{R}^{3}$, arranged in a matrix $X$. Under the action of an affine transformation, the coordinates $(x, y, z)$ are transformed into other coordinates $(\tilde{x}, \tilde{y}, \tilde{z})$ by the following procedure:

$$
\left\{\begin{array}{l}
f: \mathfrak{R}^{3} \rightarrow \mathfrak{R}^{3} \\
X(x(t), y(t), z(t)) \rightarrow f(X)=Y(x(t), y(t), z(t)) \\
Y=X(x(t), y(t), z(t)) \cdot A+B
\end{array}\right.
$$

with $A=\left(a_{i j}\right)_{i, j=1,2,3} \quad$ invertible matrix associated with the infinite, and $B$ is a vector translation in $\square^{3}$.

\section{MULTIPLE REGRESSION MODEL}

The multiple regression model is one of the most important tools in the statistics analysis [18]. It consists of studying the existing relationship between a quantitative variable $y$ and $n$ independent quantitative variables stored in a matrix $X$.

The model multiple linear or multiple regressions [19] are expressed by the following equation:

where:

$$
z=X \beta+\varepsilon
$$

- $\quad X$ is a matrix of dimension $n \times(k+1)$

- $\beta$ is the regression coefficient vector of dimension $k+1$

- $z$ is a random vector of dimension $n$.

- $\varepsilon$ is a random vector of dimension $n$ representing part residual model where $n \geq(k+1)$ and $\operatorname{Rank}(X)=k+1$.

The vectors $z$ and $\varepsilon$ undergow the following assumptions:

- $H^{(1)}: E(\varepsilon)=0$ and $E(z)=X \beta$.

- $H^{(2)}: \operatorname{cov}(\varepsilon)=\sigma^{2} I$ and $\operatorname{cov}(z)=\sigma^{2} I$ where $I$ is the unit matrix.

\subsection{Estimators}

To determine an estimator for the vector $\beta$, we use the principle of least squares. The least squares estimator is the value of the vector $\hat{\beta}$ that minimizes the following quantity : 
International Journal of Computer Science \& Information Technology (IJCSIT), Vol 3, No 1, Feb 2011

$$
Q=(z-X \beta)^{\prime}(z-X \beta)
$$

It consists of finding a vector $X \hat{\beta} \in C(X)$, the field of $X$, for which the squared of the vector $z-X \hat{\beta}$ is minimal. It is geometrically clear that this vector must be the orthogonal projection on $C(X)$, i.e: $X \hat{\beta}=X\left(X^{\prime} X\right)^{-1} X^{\prime} z \Rightarrow X^{\prime} X \hat{\beta}=X^{\prime} X\left(X^{\prime} X\right)^{-1} X^{\prime} z \Rightarrow$

\subsection{Analysis of variance}

$$
\hat{\beta}=\left(X^{\prime} X\right)^{-1} X^{\prime} z
$$

To evaluate the overall quality of the regression and know whether it should reject the initial assumptions, we do a regression analysis of variance.

The aim of the analysis of variance is to show that most of the variability of $z$ can be explained by the variability $X$ and the errors are relatively small.

In this case the regression model would be satisfactory. What is a summary?

where :

$$
S S T=\sum_{i=1}^{n}\left(z_{i}-\bar{z}\right)^{2}=\sum_{i=1}^{n}\left(z_{i}-\hat{z}_{i}\right)^{2}+\sum_{i=1}^{n}\left(\hat{z}_{i}-\bar{z}\right)^{2}=S S E+S S R
$$

- SSE : Residual Sum of Squares

- $S S R$ : Regression Sum of Squares

- SST : Total Sum of Squares

- $\hat{z}_{i}$ is the predicted value of $z_{i}$ and $\bar{z}$ is average of $z$

\subsection{Coefficient of multiple determination}

The coefficient of multiple determination is a measure of the degree of liaison between the model and observations. It measures the liaison between the independent variable $z$ and the set of explanatory variables.

It is the relation between dispersion explained by the regression (SSR) and the total dispersion (SST), i.e : it shows the proportion of variability "explained" in the total variability.

$$
R^{2}=\frac{\sum_{i=1}^{n}\left(\hat{z}_{i}-\bar{z}\right)^{2}}{\sum_{i=1}^{n}\left(z_{i}-\bar{z}\right)^{2}}=\frac{S S R}{S S T}
$$

- If $R^{2}$ is near 1 then the model is closer to reality

- If $R^{2}$ is near 0 then the model explains very little the reality. Then it must find a better model.

\subsection{Tests of hypotheses}

Hypothesis testing is a formal tool for, among other things, choosing between two hypothesis $H_{0}$ and $H_{1}$.

The hypothesis $H_{0}$ expresses that none of the $X$ variables predict $z$, i.e : 
International Journal of Computer Science \& Information Technology (IJCSIT), Vol 3, No 1, Feb 2011

$$
H_{0}: \beta_{1}=\beta_{2}=\ldots=\beta_{k}=0
$$

Versus

$$
H_{1}: \exists j \in\{1,2, \ldots, n\} \text { Such as } \beta_{j} \neq 0
$$

The significance of the multiple regression models can be tested with a variable auxiliary $F$. Indeed, under the hypothesis $H_{0}$ the statistics:

$$
F=\frac{S S R / k}{S S E / n-k-1}
$$

$F$ is distributed as $F(k, n-k-1)$. Reject $H_{0}$ if

$$
F \geq F_{\alpha}(k, n-k-1)
$$

where $F_{\alpha}(k, n-k-1)$ is the upper $\alpha$ percentage point of the (central) $F$ distribution. Alternatively, a $P$-Value can be used to carry out the test. A $P$-Value is the tail area of the central $F$ distribution beyond the calculated $F$ value, that is, the probability of exceeding the calculated $F$ value, assuming.

A $P$-Value less than $\alpha$ is equivalent to $F \geq F_{\alpha}(k, n-k-1)$.

\subsection{Verification of hypotheses $H^{(1)}$ and $H^{(2)}$}

To check the assumptions on the model, we must analyze in detail the residues. This is done graphically.

\subsubsection{Adequacy and homoscedasticity}

It consists of representing the residue as function a value predicted $\hat{z}$. If the models hypotheses are respected, it must have the independence between these two variables: the cloud does not indicate a particular structure

\subsubsection{Independence}

To test the independence, we can make a graph showing the residue as function a time or the order of observations. The analysis of independence can not be performed if the data depends on the time.

If the errors follow a specific process over time so there is a presence of autocorrelation

\section{AFFINE INVARIANT DESCRIPTION}

An invariant is a numerical quantity calculated from an object that keeps the same value when it is transformed by an element of the overall changes, i.e : let $X$ and $Y$ two objects such as:

$$
Y=X . A+B
$$

with $A=\left(a_{i j}\right)_{i, j=1,2,3}$ invertible matrix associated with the infinite, and $B$ is a vector translation in $\square^{3}$, then : 
International Journal of Computer Science \& Information Technology (IJCSIT), Vol 3, No 1, Feb 2011

$$
I=f(Y)=f(X)
$$

is an invariant where $f$ is an application defined as follows $f: \square^{p} \rightarrow \square^{q}$ with $p, q \in \square$.

Consider $z$ the regression on $X$, i.e:

We have,

$$
z=X \beta_{x}+\varepsilon_{x}
$$

$Y=X . A+B \Rightarrow Y-B=X . A \Rightarrow(Y-B) A^{-1}=X \Rightarrow z=X \beta_{x}+\varepsilon_{x}=(Y-B) A^{-1} \cdot \beta_{x}+\varepsilon_{x} \Rightarrow$ $Y \cdot A^{-1} \cdot \beta_{x}-B A^{-1} \cdot \beta_{x}+\varepsilon_{x}$.

Let : $\beta_{y}=A^{-1} \cdot \beta_{x}$ et $\varepsilon_{y}=-B A^{-1} \cdot \beta_{x}+\varepsilon_{x}$ then :

$$
z=Y \beta_{y}+\varepsilon_{y}
$$

So $z$ is regression on $Y$.

However, $Y_{c}=(Y-\bar{Y})=(A \cdot X+B-A \cdot \bar{X}-B)=A \cdot(X-\bar{X})=A \cdot X_{c} \Rightarrow$

$$
Y_{c}=A \cdot X_{c}
$$

Khowing $\hat{\beta}_{x}=\left(X_{c}^{\prime} X_{c}\right)^{-1} X_{c}^{\prime} z$ and $\hat{\beta}_{y}=\left(Y_{c}^{\prime} Y_{c}\right)^{-1} Y_{c}^{\prime} z$, according to equation (14),

$$
\begin{gathered}
\hat{\beta}_{y}=\left(Y_{c}^{\prime} Y_{c}\right)^{-1} Y_{c}^{\prime} z=\left[\left(X_{c} \cdot A\right)^{\prime}\left(X_{c} \cdot A\right)\right]^{-1}\left(X_{c} \cdot A\right)^{\prime} y=A^{-1} \cdot\left(X_{c}^{\prime} X_{c}\right)^{-1} X_{c}^{\prime} z=A^{-1} \cdot \hat{\beta}_{x} \Rightarrow \\
\hat{\beta}_{y}=A^{-1} \cdot \hat{\beta}_{x}
\end{gathered}
$$

\subsection{Calculation of the invariant $\Phi$}

Let $\Phi_{x}=f\left(X_{c}\right)=\hat{\beta}_{x}^{\prime} X_{c}$ et $\Phi_{y}=f\left(Y_{c}\right)=\hat{\beta}_{y}^{\prime} Y_{c}$ then $\Phi_{y}=\hat{\beta}_{y}^{\prime} Y_{c}=\left(A^{-1} \cdot \hat{\beta}_{x}\right)^{\prime} A \cdot X_{c}=\hat{\beta}_{x}^{\prime} X_{c}=\Phi_{x}=\Phi$

$$
\Phi_{y}=\Phi_{x}=\Phi
$$

However, let $\tilde{\beta}_{y_{i}}=1 /\left((k+1) \cdot \hat{\beta}_{\dot{y}}\right)$ if $\hat{\beta}_{y_{i}} \neq 0$ and $\tilde{\beta}_{y_{i}}=0$ else, for $i=0, \ldots, k$.

We have $\Phi=\hat{\beta}_{x}^{\prime} X_{c} \Rightarrow \tilde{\beta}_{x} \Phi=\tilde{\beta}_{x} \hat{\beta}_{x}^{\prime} X_{c}=X_{c}$ because $\tilde{\beta}_{x} \hat{\beta}_{x}^{\prime}=1$ then

So $\Phi$ is an invariant descriptor .

$$
X_{c}=\tilde{\beta}_{x} \Phi
$$

\subsection{Calculation of the invariant $S S E$}

Let $\operatorname{SSE} E_{x}=f\left(X_{c}\right)=\left(z-\hat{\beta}_{x}^{\prime} X_{c}\right)^{\prime}\left(z-\hat{\beta}_{x}^{\prime} X_{c}\right)$ et $S S E_{y}=f\left(Y_{c}\right)=\left(z-\hat{\beta}_{y}^{\prime} Y_{c}\right)^{\prime}\left(z-\hat{\beta}_{Y}^{\prime} Y_{c}\right)$ then 
$f\left(Y_{c}\right)=f\left(X_{c}\right)=(z-\Phi)^{\prime}(z-\Phi)=S S E \Rightarrow$

So SSE is an invariant.

$$
S S E=(z-\Phi)^{\prime}(z-\Phi)
$$

\subsection{Calculation of the invariant $R^{2}$}

Let $f\left(X_{c}\right)=R_{x}^{2}=\frac{S S R_{x}}{S S T}=\frac{S S T_{x}-S S E_{x}}{S S T}=1-\frac{S S E_{x}}{S S T}$ and $f\left(Y_{c}\right)=R_{y}^{2}=1-\frac{S S E_{y}}{S S T}$ then

$$
\begin{aligned}
& f\left(Y_{c}\right)=f\left(X_{c}\right)=1-\frac{(z-\Phi)^{\prime}(z-\Phi)}{S S T} \Rightarrow \\
& R^{2}=1-\frac{(z-\Phi)^{\prime}(z-\Phi)}{S S T}
\end{aligned}
$$

So $R^{2}$ is an invariant.

\section{RESULTS AND DISCUSSION}

Consider two 3D objects (origin) and (transformed) related by an affine transformation (Figures 1 and 2) and is a multiple regression model, for example $z=X \cdot a_{0}+b_{0}$ where $a_{0} \in \square^{n \times d}$ and $b_{0} \in \square^{n}$ (the choice of $a_{0}$ and $b_{0}$ are arbitrary).

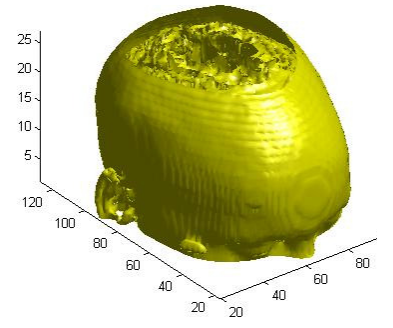

Figure 1. Origin 3D object

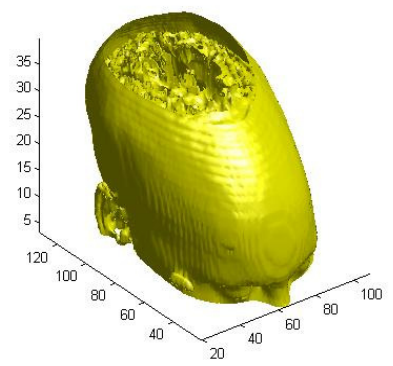

Figure 2. Transformed 3D object

To use this model to determine the invariants, it must verify its validity as multiple regressions $Y$, i.e: $\exists \beta_{y} \in \square^{k+1}$ and $\varepsilon_{y} \in \square^{n}$ such as $z=Y \beta_{y}+\varepsilon_{y}$ using the elements:

- Evaluation coefficient of multiple determination $R^{2}$

- Comparison of statistical $F$ with the quantile of Fisher $F_{\alpha}$

- Verification of hypotheses $H^{1}$ and $H^{2}$

From the above tables, we note that:

- The value of $R^{2}$ is high which indicate that there is a strong association between $z$ and $Y$.

- The value of $F$ is significative at the threshold $\alpha=0.05$, i.e: $P$-Value $\leq 0.05$ $\Rightarrow F \geq F_{\alpha}$, then we reject the hypothesis $H_{0}$. This implies the existence of significant relationship between $z$ and $Y$. 
Table 1. Coefficient of multiple determination

\begin{tabular}{|l|l|l|}
\hline Model & $R$ & $R$ Square \\
\hline 1 & 0.991 & 0.98 \\
\hline
\end{tabular}

Table 2. Analysis of variance

\begin{tabular}{|l|l|c|c|}
\hline \multicolumn{1}{|c|}{ Model } & \multicolumn{1}{c|}{ Sum of Squares } & \multicolumn{1}{c|}{$F$} & Signification , $P$-Value \\
\hline Regression & 10415.574 & 709.119 & .000 \\
Residual & 80.75 & & \\
Total & 16016.311 & & \\
\hline
\end{tabular}

In addition, according to the figures below, we note:

- The residual variability did not increase as function of the magnitude of the predicted values then there are equal variances, i.e: the errors have the same variances.

- The residues evolve in a random way that implies the absence of residuals autocorrelation.

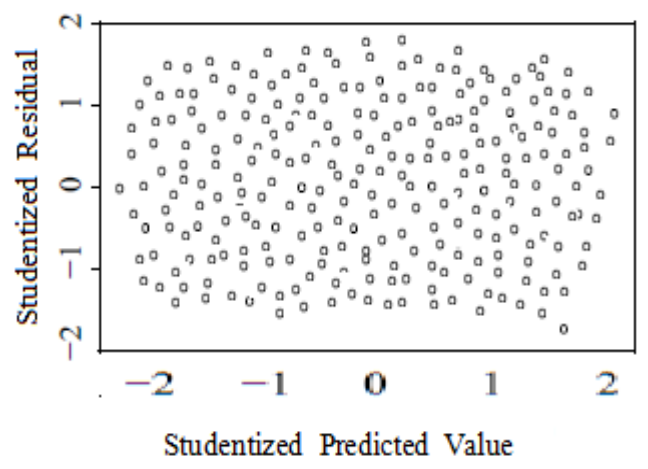

Figure 3. Graph of residual variability

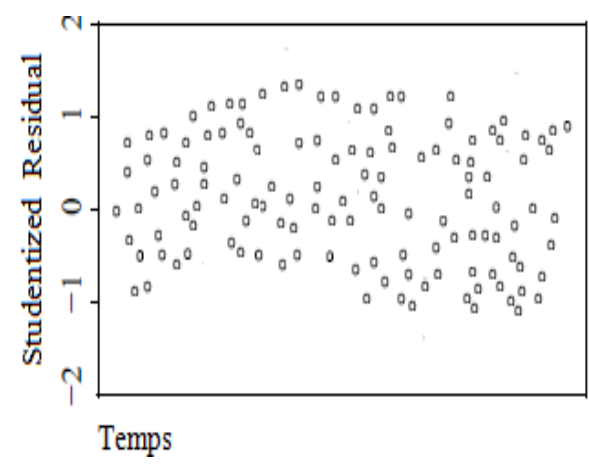

Figure 4. Graph of residual autocorrelation

Then we can say that the hypotheses $H^{1}$ and $H^{2}$ are verified. So we conclude that the multiple regression model $z$ on $Y$ is valid.

When the multiple regression models are valid, it is used to extract the following quantities: $\Phi_{x}, R_{x}$ and $S S E_{x}$ of $X$, and $\Phi_{y}, R_{y}$ and $S S E_{y}$ of $Y$

Then we calculate the following errors:

$$
\begin{aligned}
e r r_{x, y}^{1} & =\Phi_{x}-\Phi_{y} \\
e r r_{x, y}^{2} & =R_{x}-R_{y} \\
e r r_{x, y}^{3} & =S S E_{x}-S S E_{y}
\end{aligned}
$$


International Journal of Computer Science \& Information Technology (IJCSIT), Vol 3, No 1, Feb 2011

Table 3 : Values of invariants

\begin{tabular}{|l|c|c|}
\hline & Objet $X$ & Objet $Y$ \\
\hline$R_{x}$ & 0.980 \\
\hline$R_{y}$ & 0.9799 \\
\hline$e r r_{x, y}^{2}$ & 0.0001 \\
\hline$S S E_{x}$ & 80.75 \\
\hline$S S E_{y}$ & 80.7497 \\
\hline$e r r_{x, y}^{3}$ & 0.0003 \\
\hline
\end{tabular}

According to the table below, we note that the numerical quantities $R_{x}$ and $R_{y}$ are equal ( $\left.e r r_{x, y}^{2} \approx 0\right)$ and the same for $\operatorname{SSE}_{x}$ and $\operatorname{SSE}_{y}\left(\operatorname{err}_{x, y}^{3} \approx 0\right)$, this shows that these quantities are invariant.

On the other hand, according to the graphical representation of the error $\operatorname{err}_{x, y}^{1}$ (figure 7), we conclude that $\operatorname{err}_{x, y}^{1} \square 0$, this shows that the vector $\Phi$ is numerical quantity invariant $\Phi, R$ and $S S E$ are invariant, consequently $Y$ is an affine transformation of $X$.

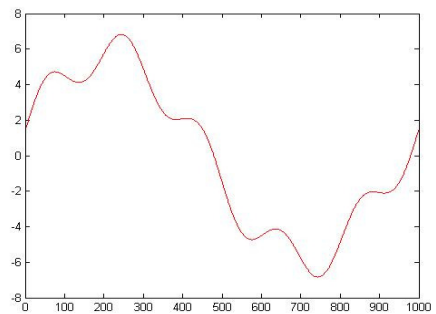

Figure 5. Descriptor of the original 3D object using MRM

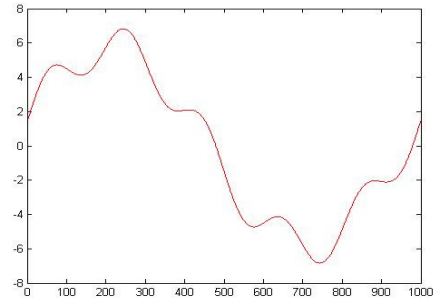

Figure 6. Descriptor of the transformed 3D object using MRM

According to the figures below we can see that the error ${ }^{4}$ - corresponds to the difference between the invariants of the object origin and its transformation by an affine transformationcalculated by our method (figure 7) is smaller than those obtained by the methods of Fourier transform and Moments (figures 8 and 9).

In addition, the computing time of our method is less than the method of Fourier transform and Moments. This leads us to conclude that our method applied to these objects is more efficient than Fourier transform and Moments.

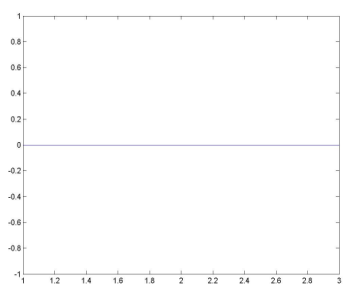

Figure 7.Error representation by proposed method

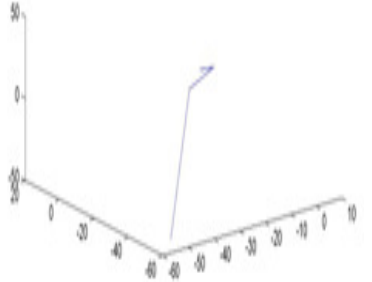

Figure 8. Error ${ }^{4}$ representation by method Moments

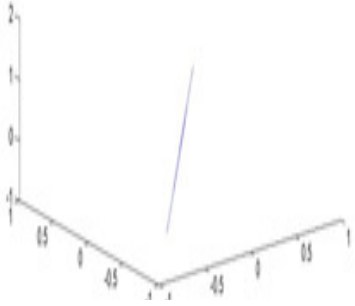

Figure 9. Error ${ }^{4}$ representation by Fourier transform 


\section{CONCLUSION}

The size of 3D data stored around the Web has become bigger. Therefore the development of recognition applications and retrieval systems of 3D models is important. This paper advocate the use of affine invariants to describe 3D objects.

In this work we have presented an invariant method of 3D objects based on multiple regression model. This method consists in first time of proposing a regression model that will be applied to both objects after its validation and use it to define the invariants using its parameters. The simulation results were presented. They were generally satisfactory and show the validity of our method.

In addition, we compared our method with those of Fourier transform and Moments. The results of this comparison are favorable to our method

\section{REFERENCES}

[1] Ahmed El Oirrak, Mohamed Daoudi, Driss Aboutajdine: Affine invariant descriptors for color images using Fourier series. Pattern Recognition Letters 24(9-10): 1339-1348 (2003).

[2] Ahmed El Oirrak, Mohamed Daoudi, Driss Aboutajdine: Estimation of general 2D affine motion using Fourier descriptors. Pattern Recognition 35(1): 223-228 (2002).

[3] Ahmed El Oirrak, Mohamed Daoudi, Driss Aboutajdine: Affine invariant descriptors using Fourier series. Pattern Recognition Letters 23(10): 1109-1118 (2002).

[4] T. Filali Ansary, M. Daoudi, and J.P. Vandeborre. A bayesian 3d search engine using adaptive views clustering. IEEE Transaction on Multimedia, 9:78-88, 2007.

[5] A. Lmaati, A. El Oirrak, and M. N. Kaddioui. A 3d search engine based on 3d curve analysis. SIViP: Signal, Image and Video processing, 2009.

[6] E. A. Lmaati, A. El Oirrak, and M. N. Kaddioui. 3d model retrieval based on 3d discrete cosine transform. IAJIT, International Arab Journal of Information Technology, 2010.

[7] P. Shilane, M. Kazhdan, P. Min, and T. Funkhouser. The princeton shape benchmark. In Shape Modeling International, pages 345-352, 2004.

[8] F. Ghorbel, M. Chakchouk, et S. Derrode. Comparaison de descripteurs locaux-globaux pour l'indexation d'images à niveaux de gris. Dans Compression et Représentation des Signaux Audiovisuels (CORESA' 00), Poitiers, France, 19-20 octobre 2000.

[9] T. Zaharia et F. Prêteux. 3D versus 2D/3D shape descriptors : A comparative study. Dans SPIE Conference on Image Processing : Algorithms and Systems III, volume 5298, San Jose, USA, 18.22 january 2004.

[10] D.V. Vranic. An improvement of rotation invariant 3d-shape descriptor based on functions on concentric spheres. In IEEE International Conference on Image Processing (ICIP03), pages 757760, 2003.

[11] V. Vranic and D. Saupe. 3d model retrieval with spherical harmonics and moments. In B. Radig and S. Florczyk (Eds.), DAGM 2001, pages 392-397, Munich, Germany, 2001.

[12] C. Zhang et T. Chen. Ef_cient feature extraction for 2D/3D objects in mesh representation. Dans International Conference on Image Processing (ICIP'01), Thessaloniki, Greece, 7.10 October 2001.

[13] M. Novotni et R. Klein. 3D Zernike descriptors for content based shape retrieval. Dans ACM Symposium on Solid Modeling and Applications, pages 216.225, Seattle, USA, 16.20 June 2003.

[14] D. Vranic et J. Saupe, D.and Richter. Tools for 3dobject retrieval : Karhune-loeve transform and spherical harmonics. Dans IEEE Workshop Multimedia Signal Processing, Cannes, France, 3.5 October 2001.

[15] V. Vranic and D. Saupe. 3d model retrieval with spherical harmonics and moments. In B. Radig and S. Florczyk (Eds.), DAGM 2001, pages 392-397, Munich, Germany, 2001.

[16] F. Prêteux T. Zaharia. Indexation de maillages 3D par descripteurs de forme. Dans Reconnaissance des Formes et Intelligence Arti_cielle (RFIA'2002), pages 48.57, Angers, France, 8.10 janvier 2002. 
International Journal of Computer Science \& Information Technology (IJCSIT), Vol 3, No 1, Feb 2011

[17] C. Zhang and T. Chen. Efficient feature extraction for $2 d / 3 d$ objects in mesh representation. In IEEE International Conference on Image Processing ICIP, pages 935-938, Lausanne, Switzerland, 2000.

[18] Bouroche, J.M. et G. Saporta (2006), L'analyse de données, Presses Universitaires de France, Collection Que sais-je ?, 9ème édition, 128p., 2006.

[19] Saporta, G. (2006), Probabilités, analyse des données et statistiques, Technip, 2ème édition, 622 p., 2006.

Authors

Elhachloufi Mostafa is a researcher at the Department of Computer Science, Faculty of Science Samlalia, Cady Ayyad University, Marrakech, Morocco. His current research interests are multimedia information retrieval, virtual reality, new media, computer graphics and multimedia databases.

Ahmed El Oirrak received the CEUS and 3rd Cycle thesis both in computer science from the Faculty of Science, Mohammed V University, Rabat, Morocco, in 1996 and 1999, respectively. He joined Cadi Ayyad University, Marrakech, Morocco, in 1999, first as an assistant professor, and received the Doctorate in signal processing from the Mohammed V University, Rabat, Morocco, in 2001. $\mathrm{He}$ is presently an associate professor with the Faculty of the Sciences of Marrakech Semlalia. His research interests include image processing, pattern recognition, and their applications. He is the author of more than 20 publications.
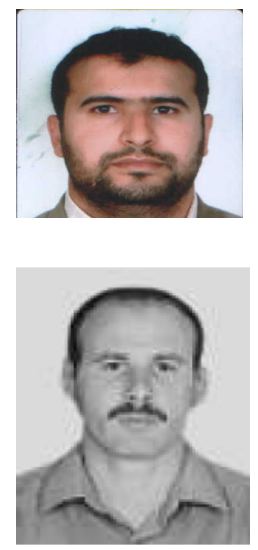

Driss Aboutajdine obtained the license in physics in 1976, the Certificate of Advanced Studies in Computer Science and Signal Processing in 1977, 3rd cycle diploma and doctorate in signal processing and image from the University Mohammed V-Agdal respectively in 1980 and 1985 . He is currently a professor at the Faculty of Sciences of Rabat, where he directs the Laboratory for Research in Computing and Telecommunications. Since 2001, he was National Coordinator of the ICT center of excellence "Sciences and Technologies of Information and Communication", which covers more than 30 laboratories and university research teams and several industry partners. He is a member of IEEE since 1988 and senior member since 1999. He is also a member of ACM since 2004. Driss Aboutajdine was repeatedly professor or visiting scholar at several institutions of higher education and research in Europe which Suptelecom Paris, Université Bordeaux I, University of Rouen, Nantes University and the Polytechnic University of Catalonia and North America, the University of Southern Massachusetts EMS and MIT and Stanford University.

Mohammed Najib Kaddioui is a full professor of computer science at the Department of Computer Science, Faculty of Science Samlalia, Caddy Ayyad University, Marrakech, Morocco. His major field of study is information processing and management, and computer graphics.
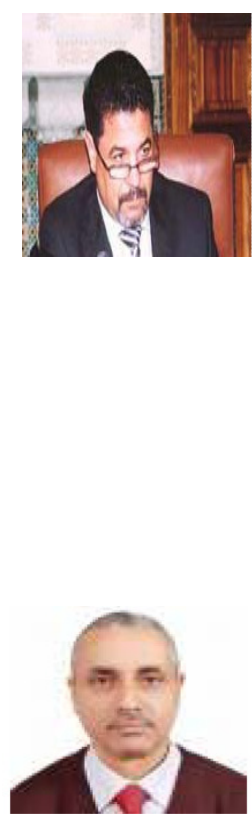